\title{
A comprehensive guidelines-based approach reduces cardiovascular risk in everyday practice: the VARO study
}

Tomáš Štulc ${ }^{1}$, Věra Lánská2 ${ }^{2}$ Michaela Šnejdrlová ${ }^{1}$, Michal Vrablík $^{1}$, Martina Prusíková ${ }^{1}$, Richard Češka ${ }^{1}$

\author{
${ }^{1} 3^{\text {rd }}$ Department of Internal Medicine, $1^{\text {st }}$ Faculty of Medicine, Charles University, \\ Prague, Czech Republic \\ 2Division of Professional Activities, Quality Assurance and Controlling, Institute \\ for Clinical and Experimental Medicine, Prague, Czech Republic
}

Submitted: 7 October 2015

Accepted: 24 October 2016

Arch Med Sci 2017; 13, 4: 705-710

DOI: https://doi.org/10.5114/aoms.2016.64865

Copyright $\odot 2016$ Termedia \& Banach

\section{Abstract}

Introduction: The aim of study was to investigate the possibility of cardiovascular risk improvement through systematic identification of high-risk individuals and treatment in accordance with current guidelines using modern therapy in daily clinical practice.

Material and methods: Two hundred and sixty-three physicians participated in the study. The physicians were asked to screen for cardiovascular risk factors in patients presenting with unrelated problems and to re-evaluate the attainment of treatment goals in those with already known risk factors. Each physician enrolled up to 20 consecutive patients with hypertension and/or hyperlipidemia. A total of 3015 patients were included. Cardiovascular risk was assessed using the SCORE system. Risk factors were treated in accordance with current national guidelines. The therapy of hyperlipidemia and hypertension was preferentially based on rosuvastatin, amlodipine and valsartan. Further medication was at the discretion of the attending physician. Patients were examined at baseline and after 3 and 6 months.

Results: The principal result is that global cardiovascular risk decreased by $35 \%$ (from $8.9 \pm 6.4$ to $5.9 \pm 4.4, p<0.001$ ). Systolic and diastolic blood pressure decreased by $12.5 \%$ (from $152 \pm 18$ to $133 \pm 11, p<0.001$ ) and $11.4 \%$ (from $88 \pm 11$ to $78 \pm 7, p<0.001$ ). The level of total cholesterol decreased $21 \%$ (from $6.3 \pm 1.2$ to $5.0 \pm 0.9, p<0.001$ ) and the concentration of LDL-C decreased $28 \%$ (from $3.9 \pm 1.1$ to $2.8 \pm 0.8, p<0.001$ ). HDL-C increased by $7 \%$ (from $1.43 \pm 0.58$ to $1.53 \pm 0.56, p<0.001$ ) and triglycerides decreased by $25 \%$ (from $2.4 \pm 1.3$ to $1.8 \pm 0.9, p<0.001$ ). Blood pressure and LDL-C target values were reached in $68 \%$ and $34 \%$ of patients, respectively.

Conclusions: The VARO study demonstrates that in daily practice settings, both individual risk factors and global cardiovascular risk are significantly improved through the systematic identification of high-risk individuals and their treatment in accordance with current guidelines using modern pharmacotherapy.

Key words: global cardiovascular risk, hypertension, dyslipidemia, cardiovascular risk reduction, guidelines implementation.

\section{Introduction}

Prevention of cardiovascular disease is crucial for reducing cardiovascular risk in the population. A number of studies have demonstrated the possibility of reducing cardiovascular risk (CVR) through the therapy of cardiovascular risk factors [1-11]. These results, however, may appear

\author{
Corresponding author: \\ Tomáš Štulc MD, PhD \\ $3^{\text {rd }}$ Department \\ of Internal Medicine \\ U Nemocnice 1 \\ 12821 Praha 2 \\ Czech Republic \\ Phone: +420-22496 2918 \\ Fax: +420-224919780 \\ E-mail: tstulc@lf1.cuni.cz
}


difficult to apply to a wide range of patients who are often substantially different from carefully selected participants of the respective trials. Also, trial participants are treated with precisely defined therapies, while, in routine practice, patients typically receive multiple medications in diverse dosing regimens, raising the issues of side effects, drug interactions, and patient compliance with therapy [12]. In addition, there are other barriers to practical application of the scientific knowledge, including previous expertise of the doctor, personal preferences and attitudes of doctors and patients, insufficient knowledge, lack of time, and economic factors [13-17]. In line with this, control of cardiovascular risk factors in the general population is far from satisfactory, and therapies with proven efficacy are underused [18, 19].

Obviously, therefore, translating the knowledge gained in clinical trials into clinical practice is less self-evident than expected, and the problem of application of research knowledge deserves attention. However, compared to the large body of clinical trial data, there is a paucity of research addressing the issue of effectively translating scientific evidence into routine clinical practice [20-26]. The aim of the VARO study (Valsartan Amlodipine and ROsuvastatin for global cardiovascular risk decrease in daily practice) was to assess the possibility of CVR improvement through the systematic identification and subsequent treatment of high-risk individuals using guideline-based therapy in clinical practice.

\section{Material and methods}

\section{Design of the study}

Physicians who are routinely involved in treatment of cardiovascular risk factors on an outpatient basis (general practitioners, internists, cardiologists and diabetologists) were offered to participate in the study. The information about the study was disseminated through e-mail and during seminars and symposia. Two hundred and sixty-three physicians, of whom $68 \%$ were general practitioners, $26 \%$ internists, $5 \%$ cardiologists, and $1 \%$ diabetologists, participated in the study. The study was approved by the State Institute for Drug Control.

The physicians were asked to screen for cardiovascular risk factors in all patients who sought their consultation, regardless of the presenting problem. Individuals with hypertension and/or dyslipidemia (either established or newly diagnosed during the initial visit) were enrolled in the study. Each physician enrolled up to 20 consecutive patients. Dyslipidemia was defined as total cholesterol (TC) $>5.0 \mathrm{mmol} / \mathrm{l}$ or triglycerides (TG) > $1.7 \mathrm{mmol} / \mathrm{l}$ or high-density lipoprotein cholesterol (HDL-C)
$<1.0 \mathrm{mmol} / \mathrm{l}$ (males)/ < $1.2 \mathrm{mmol} / \mathrm{l}$ (females) or use of lipid-lowering drugs. Hypertension was defined as systolic blood pressure $>140 \mathrm{~mm} \mathrm{Hg}$ or diastolic blood pressure $>90 \mathrm{~mm} \mathrm{Hg}$ or use of antihypertensive drugs. History of cardiovascular disease (CVD) was defined as a history of coronary heart disease, heart failure, stroke, or peripheral vascular disease. Smoking was defined as having smoked within the last 4 weeks.

Patients were examined at baseline and again after 3 and 6 months. During each visit, clinical and laboratory examinations were performed and global CVR was assessed. Based on these examinations, the attainment of treatment goals was evaluated and the therapy for risk factors was adjusted accordingly.

\section{Examinations}

Biochemical tests were performed at local laboratories using automated analyzer methods. All patients were examined for total cholesterol (TC), low-density lipoprotein-cholesterol (LDL-C), high-density lipoprotein-cholesterol (HDL-C), triglycerides (TG), glycemia and safety laboratory tests, including serum transaminases, creatinine and creatine kinase. A subgroup of patients was examined for apolipoproteins apo A-I, apo B and lipoprotein(a) (Lp(a)). Global CVR (expressed as a 10-year risk of fatal cardiovascular disease) was assessed using the SCORE risk charts (charts specific for the Czech Republic were used) [27].

\section{Treatments}

Risk factors were treated in accordance with current national guidelines, which are based on European guidelines on cardiovascular disease prevention [28]. In addition, lifestyle measures were recommended to all patients. Hyperlipidemia and hypertension therapy was preferentially based on rosuvastatin, amlodipine and valsartan, but other drugs prescribed by the attending physician were considered to be acceptable. Any further medication was at the discretion of the attending physician.

\section{End points}

The primary outcome was change in cardiovascular risk. Secondary outcomes included changes in serum lipids and blood pressure. In addition, changes in blood glucose, apolipoproteins, body mass index (BMI) and waist circumference were evaluated.

\section{Statistical analysis}

Continuous variables were described by mean and standard deviation and discrete ones by ab- 
solute and relative frequencies. Time changes for continuous variables were evaluated by ANOVA with repeated measures and pairwise comparisons performed by the contrast method. McNemar's test, with Bonferroni correction for significance levels, was used for discrete variables. All tests were two-sided and $p<0.05$ was considered to be significant. All calculations were done by SYSTAT software version 13 (Systat Software, Inc, USA).

\section{Results}

A total of 3015 patients were included and 2932 completed the study. Baseline characteristics of the patients are shown in Table I and the main outcomes of the study are shown in Table II. Patients who dropped out did not differ from those who completed the study. The most significant result is the profound decrease in global CV risk which became evident after the second visit and a further drop by the third visit (by $21 \%$ and $35 \%$, respectively). The improvement in the SCORE risk estimate was driven by the simultaneous decrease in systolic blood pressure and total cholesterol concentration. In addition, a highly significant improvement in diastolic blood pressure and in other lipid values was attained. The decrease in LDL-C was paralleled by a decrease in apoB concentration. ApoA concentrations also decreased, de-
Table I. Baseline characteristics of study subjects

\begin{tabular}{|lc|}
\hline Parameter & Value \\
\hline Number & 2932 \\
\hline Male/female (\%) & $52 / 48$ \\
\hline Age [years] & $61 \pm 10.5$ \\
\hline BMI [kg/m $\left.{ }^{2}\right]$ & $29.4 \pm 4.3$ \\
\hline History of CVD & $48.2 \%$ \\
\hline Hypertension & $69.5 \%$ \\
\hline Diabetes mellitus & $20 \%$ \\
\hline Smoking (\%) & 17 \\
\hline
\end{tabular}

$B M I$ - body mass index, CVD - cardiovascular disease.

spite an increase in $\mathrm{HDL}-\mathrm{C}$, while concentrations of $L p(a)$ remained unchanged. Concerning other variables, there was a decrease in blood glucose and a small, but still significant, decrease in body weight, BMI and waist circumference.

In terms of target values, significantly more patients attained target values for blood pressure and LDL-C during the second and third visits compared to the baseline $(19.5 \%, 44.6 \%$ and $67.8 \%$ for blood pressure and $10.6 \%, 25.6 \%, 34.3 \%$ for LDL-C, at the first, second and third visits respectively). The study treatments were well tolerated. Creatine kinase levels increased moderately, and there was no increase in liver or renal tests (Table II).

Table II. Main outcomes of study

\begin{tabular}{|c|c|c|c|c|c|}
\hline Parameter & Visit 1 & Visit 2 & $\%$ & Visit 3 & $\%$ \\
\hline Global CVR* & $8.9 \pm 6.4$ & $6.7 \pm 4.8$ & -24.7 & $5.9 \pm 4.4$ & -33.7 \\
\hline $\mathrm{SBP}[\mathrm{mm} \mathrm{Hg}]$ & $152 \pm 18$ & $139 \pm 13$ & -8.6 & $133 \pm 11$ & -12.5 \\
\hline $\mathrm{DBP}[\mathrm{mm} \mathrm{Hg}]$ & $88 \pm 11$ & $81 \pm 8$ & -8.0 & $78 \pm 7$ & -11.4 \\
\hline $\mathrm{TC}[\mathrm{mmol} / \mathrm{l}]$ & $6.3 \pm 1.2$ & $5.4 \pm 1.0$ & -14.3 & $5.0 \pm 0.9$ & -20.6 \\
\hline LDL-C [mmol/l] & $3.9 \pm 1.1$ & $3.1 \pm 0.9$ & -20.5 & $2.8 \pm 0.8$ & -28.2 \\
\hline $\mathrm{HDL}-\mathrm{C}[\mathrm{mmol} / \mathrm{l}]$ & $1.43 \pm 0.58$ & $1.49 \pm 0.53$ & +4.2 & $1.53 \pm 0.56$ & +7.0 \\
\hline TG [mmol/ll] & $2.4 \pm 1.3$ & $1.9 \pm 0.9$ & -20.8 & $1.8 \pm 0.9$ & -25.0 \\
\hline ApoB $[\mathrm{g} / \mathrm{l}]^{\star *}$ & $1.21 \pm 0.43$ & $1.02 \pm 0.30$ & -15.7 & $0.97 \pm 0.38$ & -19.8 \\
\hline ApoA-I [g/l] $]^{* *}$ & $1.44 \pm 0.43$ & $1.30 \pm 0.40$ & -9.7 & $1.23 \pm 0.34$ & -14.6 \\
\hline $\operatorname{Lp}(\mathrm{a})[\mathrm{g} / \mathrm{l}]^{\star \star}$ & $0.23 \pm 0.15$ & $0.22 \pm 0.12^{\dagger}$ & -4.3 & $0.21 \pm 0.09^{t \dagger}$ & -8.7 \\
\hline Weight [kg] & $86 \pm 15$ & $85 \pm 14$ & -1.2 & $84 \pm 14$ & -2.3 \\
\hline BMI $\left[\mathrm{kg} / \mathrm{m}^{2}\right]$ & $29.4 \pm 4.3$ & $29.0 \pm 4.2$ & -1.4 & $28.7 \pm 4.2$ & -2.4 \\
\hline Waist circumference $[\mathrm{cm}]^{\star * *}$ & $97 \pm 14$ & $96 \pm 13$ & -0.8 & $96 \pm 13$ & -1.6 \\
\hline Glucose $[\mathrm{mmol} / \mathrm{l}]$ & $6.1 \pm 1.6$ & $5.9 \pm 1.3$ & -3.3 & $5.7 \pm 1.3$ & -6.6 \\
\hline CK $[\mu k a t / l]$ & $2.05 \pm 1.08$ & $2.26 \pm 1.20$ & +10.2 & $2.33 \pm 1.20$ & +13.7 \\
\hline
\end{tabular}

Global CVR is expressed as the 10-year risk of fatal cardiovascular disease. CVR - cardiovascular risk, SBP-systolic blood pressure, DBP - diastolic blood pressure, BMI - body mass index, CK - creatine kinase. *Global CVR was calculated only in subjects without a history of cardiovascular disease (1518 patients); ${ }^{* *}$ apolipoprotein and $L p(a)$ concentrations were examined in a subgroup of 236 subjects; ${ }^{* * *}$ waist circumference was measured standing, just above the upper hip bone, at the end of normal expiration. ${ }^{\dagger}$ non-significant visit 2 vs. visit 1 ; ${ }^{+1}$ non-significant visit 2 vs. visit 1; all remaining differences between visit 2/visit 1 and visit 3/visit 1 were significant at $p<0.001$. 


\section{Discussion}

The VARO study demonstrated substantial improvement of the global cardiovascular risk estimate and several individual risk factors in the daily clinical practice settings. The global CVR, as assessed using the SCORE risk prediction model, decreased by one third. This goal was achieved through the improved identification of high-risk individuals and greater adherence to current treatment guidelines and modern drug therapy.

At first glance, the VARO study may resemble conventional trials of the efficacy of antihypertensive and hypolipidemic drugs, but the focus and importance of our study are quite different. The efficacy of neither drugs nor drug combinations was studied, but instead the effect of a complex approach to the identification and treatment of high-risk individuals in daily practice was explored. Doctors were simply asked to assess CVR, check for the attainment of blood pressure and lipid targets, and treat patients accordingly. For therapy, modern drugs were recommended but were not obligatory. No supervision or feedback was provided to doctors, and no aspects of patient assessment and treatment were evaluated or commented on during the study. The VARO study did not measure the effect of any therapy under controlled condition; instead it explored the feasibility and effect of the implementation of a guidelines-based approach in real-life settings.

Putting best practices into practice is crucial for reducing cardiovascular risk in the population. There are, at present, a variety of effective drugs available to achieve target values in most patients; however, there remains the problem that the available treatment options are underused [18, 28-30]. EUROASPIRE studies have shown that even in patients with a history of cardiovascular disease, who are at the highest risk of future events, appropriate blood pressure and cholesterol levels are achieved in less than half of all patients [18]. Therefore, the issue of implementing guidelines for cardiovascular disease prevention demands attention. Nevertheless, there are only a few studies that examine the effectiveness of various methods to improve adherence to the guidelines [20-25, 31, 32]. In this context, the VARO study demonstrates that a relatively simple approach may translate into an unexpectedly large reduction in cardiovascular risk. Our results parallel those obtained by Greek authors, who evaluated strategies to improve therapy of hyperlipidemia [24] or hypertension [25]. Compared to our study, those studies used a more stringent treatment protocol and demonstrated even greater reduction in the cardiovascular risk score.

It may seem difficult to identify the factors contributing to the favorable results of our study.
Generally speaking, the doctors were asked to do what they should have done anyway, and what they would really have done in many patients. There was little new information in the simple risk evaluation and treatment recommendations. The most important and seemingly simple point is to remind doctors to do what they are already used to doing more consistently. However, reminding itself may not necessarily result in better outcomes. In a study by German authors, education of primary care physicians in cardiovascular prevention did not improve prescription of statins when compared to a group receiving no education [20], while education of physicians in hospitals resulted in improvement in cardiovascular risk prevention [33]. It would be therefore a step forward to identify specific factors that could have contributed most to the improvement observed in our study.

Most important, in our opinion, appears to be consistently evaluating and re-evaluating cardiovascular risk in all patients. It is clear that, in current practice, this evaluation is not routinely performed for people who have visited a doctor for something other than cardiovascular disease. The fact that the data associated with cardiovascular risk, blood pressure and serum lipids had to be recorded on the patient charts and explicitly compared with the desired target values lowered the likelihood of the doctor overlooking or underestimating the result. Also, a method of cardiovascular risk assessment could play a role. Physicians often use subjective assessment rather than risk charts or calculators [14], which often results in underestimating the risk in patients of older age groups. Consistent use of SCORE risk charts in our study could help to identify more patients at risk who require treatment. Finally, recommending the use of modern drugs to treat hypertension and dyslipidemia could eventually support the change in therapy in patients who have been treated with older, less effective therapies.

Obviously, our study has many limitations, especially if compared to conventional clinical trials. The lack of a control group appears most important. On the other hand, the design of the study precludes including a control group, because the screening itself (i.e. systematic assessment of CVR) was considered an important part of the complex approach to risk reduction. Also, there is no detailed information about the quality of dietary and lifestyle intervention or the changes achieved by the patients, which are all difficult to assess reliably. However, as noted above, the VARO study did not measure the effect of any particular therapy under controlled conditions, but rather assessed the overall effect of the complex approach to cardiovascular disease prevention.

The question remains whether the changes demonstrated in our study will persist even after 
treatment. If we assume that the primary reason for the improved treatment was the attention given to patients, it is likely that the transition to normal controls, without the prescribed frequency and scope of the visits, will lead to some degree of worsening of results. An evaluation of the effects more than a year following the end of the study will be the subject of further research.

\section{Acknowledgments}

The project was supported bygrants NT 12217-5/ 2011 and NT 14152-3/2013 from IGA MZ CR and by an educational grant from Teva Pharmaceuticals CR.

\section{Conflict of interests}

$\mathrm{RC}$ and MV have received consultation honoraria from Teva Pharmaceuticals CR.

\section{References}

1. Sever PS, Dahlöf B, Poulter NR, et al. Prevention of coronary and stroke events with atorvastatin in hypertensive patients who have average or lower-than-average cholesterol concentrations, in the Anglo-Scandinavian Cardiac Outcomes Trial--Lipid Lowering Arm (ASCOT-LLA): a multicentre randomised controlled trial. Lancet 2003; 361: 1149-58.

2. Hsia J, MacFadyen JG, Monyak J, Ridker PM. Cardiovascular event reduction and adverse events among subjects attaining low-density lipoprotein cholesterol $<50 \mathrm{mg} / \mathrm{dl}$ with rosuvastatin. The JUPITER trial (Justification for the Use of Statins in Prevention: an Intervention Trial Evaluating Rosuvastatin). J Am Coll Cardiol 2011; 57: 1666-75.

3. Teo K, Yusuf S, Sleight P, et al. Rationale, design, and baseline characteristics of 2 large, simple, randomized trials evaluating telmisartan, ramipril, and their combination in high-risk patients: the Ongoing Telmisartan Alone and in Combination with Ramipril Global Endpoint Trial/ Telmisartan Randomized Assessment Study in ACE Intolerant Subjects with Cardiovascular Disease (ONTARGET/ TRANSCEND) trials. Am Heart J 2004; 148: 52-61.

4. Cholesterol Treatment Trialists' (CTT) Collaboration. Efficacy and safety of more intensive lowering of LDL cholesterol: a meta-analysis of data from 170,000 participants in 26 randomised trials. Lancet 2010; 376: 1670-81.

5. Czernichow S, Zanchetti A, Turnbull F, et al. Blood Pressure Lowering Treatment Trialists' Collaboration. The effects of blood pressure reduction and of different blood pressure-lowering regimens on major cardiovascular events according to baseline blood pressure: meta-analysis of randomized trials. J Hypertens 2011; 29: 4-16.

6. Perk J, De Backer G, Gohlke H, et al. European Guidelines on cardiovascular disease prevention in clinical practice (version 2012). Eur Heart J 2012; 33: 1635-701.

7. Serban MC, Banach M, Mikhailidis DP. Clinical implications of the IMPROVE-IT trial in the light of current and future lipid-lowering treatment options. Expert Opin Pharmacother 2016; 17: 69-80.

8. Aronow WS. Lipid-lowering therapy in older persons. Arch Med Sci 2015; 11: 43-56.
9. Myśliwiec M, Walczak M, Małecka-Tendera E, et al. Management of familial hypercholesterolemia in children and adolescents. Position paper of the Polish Lipid Expert Forum. J Clin Lipidol 2014; 8: 173-80.

10. Banach M, Aronow WS, Serban C, et al. Lipids, blood pressure and kidney update 2014. Pharmacol Res 2015; 95-96: 111-25.

11. Colantonio LD, Baber U, Banach M, et al. Contrasting cholesterol management guidelines for adults with CKD. J Am Soc Nephrol 2015; 26: 1173-80.

12. Nichol MB, Knight TK, Wu J, et al. Transition probabilities and predictors of adherence in a California Medicaid population using antihypertensive and lipid-lowering medications. Value Health 2009; 12: 544-50.

13. Graham IM, Stewart M, Hertog MG. Factors impeding the implementation of cardiovascular prevention guidelines: findings from a survey conducted by the European Society of Cardiology. Eur J Cardiovasc Prev Rehabil 2006; 13: 839-45.

14. Grol R, Grimshaw J. From best evidence to best practice: effective implementation of change in patients' care. Lancet 2003; 362: 1225-30.

15. van Steenkiste B, van der Weijden T, Timmermans D, Vaes J, Stoffers J, Grol R. Patients' ideas, fears and expectations of their coronary risk: barriers for primary prevention. Patient Educ Couns 2004; 55: 301-7.

16. Zwolsman S, te Pas E, Hooft L, Wieringa-de Waard M, van Dijk N. Barriers to GPs' use of evidence-based medicine: a systematic review. Br J Gen Pract 2012; 62: e511-21.

17. Eichler K, Zoller M, Tschudi P, Steurer J. Barriers to apply cardiovascular prediction rules in primary care: a postal survey. BMC Fam Pract 2007; 8: 1.

18. Kotseva K, Wood D, De Backer G, De Bacquer D, Pyorala K, Keil U. Cardiovascular prevention guidelines in daily practice: a comparison of EUROASPIRE I, II, and III surveys in eight European countries. Lancet 2009; 373: 929-40.

19. Cífková R, Skodová Z, Bruthans J, et al. Longitudinal trends in major cardiovascular risk factors in the Czech population between 1985 and 2007/8. Czech MONICA and Czech post-MONICA. Atherosclerosis 2010; 211: 676-81.

20. Keller H, Hirsch O, Kaufmann-Kolle P, et al. Evaluating an implementation strategy in cardiovascular prevention to improve prescribing of statins in Germany: an intention to treat analysis. BMC Public Health 2013; 13: 623.

21. van de Steeg-van Gompel CH, Wensing M, De Smet PA. Implementation of a pharmacist-led intervention to enhance statin prescribing for secondary prevention in primary care: a cluster randomized trial. Eur J Prev Cardiol 2012; 13: 169-76.

22. Smidth M, Christensen MB, Olesen F, Vedsted P. Developing an active implementation model for a chronic disease management program. Int J Integr Care 2013; 13: e020.

23. Bernhardsson S, Larsson ME, Eggertsen R, et al. Evaluation of a tailored, multi-component intervention for implementation of evidence-based clinical practice guidelines in primary care physical therapy: a non-randomized controlled trial. BMC Health Serv Res 2014; 14: 105.

24. Hatzitolios Al, Athyros VG, Karagiannis A, et al. IMPROVE Collaborative Group. Implementation of strategy for the management of overt dyslipidemia: the IMPROVE-dyslipidemia study. Int J Cardiol 2009; 134: 322-9.

25. Karagiannis A, Hatzitolios Al, Athyros VG, et al. Implementation of guidelines for the management of arterial hypertension. The IMPULSION study. Open Cardiovasc Med J 2009; 3: 26-34. 
26. Reiner Z, Tedeschi-Reiner E. Prevalence and types of persistent dyslipidemia in patients treated with statins. Croat Med J 2013; 54: 339-45.

27. Cifkova R, Byma S, Ceska R, et al. Cardiovascular disease prevention in adults. Joint guidelines of the Czech national societies. Cor Vasa 2005; 47 Suppl: 3-14.

28. Perk J, De Backer G, Gohlke H, et al. European guidelines on cardiovascular disease prevention in clinical practice (version 2012). Eur Heart J 2012; 33: 1635-701.

29. Ghandehari H, Kamal-Bahl S, Wong ND. Prevalence and extent of dyslipidemia and recommended lipid levels in US adults with and without cardiovascular comorbidities: the National Health and Nutrition Examination Survey 2003-2004. Am Heart J 2008; 156: 112-9.

30. Berthold HK, Gouni-Berthold I, Böhm M, Krone W, Bestehorn KP. Patterns and predictors of statin prescription in patients with type 2 diabetes. Cardiovasc Diabetol 2009; 8: 25.

31. Dorobantu M, Tautu OF, Darabont R, et al. Objectives and methodology of Romanian SEPHAR II Survey. Project for comparing the prevalence and control of cardiovascular risk factors in two East-European countries: Romania and Poland. Arch Med Sci 2015; 11: 715-23.

32. Swarowska M, Burkot J, Janowska A, et al. Improvement of survival in Polish stroke patients is related to reduced stroke severity and better control of risk factors: the Krakow Stroke Database. Arch Med Sci 2016; 12: 552-5.

33. Kawecka-Jaszcz K, Jankowsk P, Pajakb A, et al. Cracovian program for secondary prevention of ischaemic heart disease. Secondary prevention of ischaemic heart disease during hospitalisation in 1996/1997 and 1998/1999. Eur Heart J Suppl 2004; 6: J47-52. 